\title{
Educar desde las emociones: panorama docente en la educación chilena
}

\author{
Educating from the emotions: a teaching landscape in Chilean education
}

Pablo Cornejo Asmussen ${ }^{1 *}$, Andrés Cabezas Corcione²

\begin{abstract}
RESUMEN
Los ambientes de aprendizaje se sustentan en las relaciones humanas, las actividades interpersonales que se establecen, el abordaje de situaciones de conflicto, la promoción de vínculos saludables, el ejercicio de la autoridad por parte del docente, la lectura emocional, el reconocimiento del otro más allá de lo cognitivo, la capacidad de escucha y el valor de empatizar con distintas situaciones del alumnado que afectan el contexto escolar, dependen, entre otras cosas, de las competencias socioemocionales del adulto que ejerce el rol pedagógico, que se relacionan con la motivación de los estudiantes frente al aprendizaje y rendimiento académico. El objetivo del manuscrito apunta a dar una vista panorámica a la situación educativa actual y la salud mental docente además de establecer la importancia de la educación emocional y la psicología positiva como paradigma en el ámbito educativo. Finalmente se discute la implicancia que posee la educación emocional y la psicología positiva en el contexto educacional.
\end{abstract}

Palabras clave: Educación emocional, competencias socioemocionales, psicología positiva.

\begin{abstract}
Educational settings are sustained by human relationships, established interpersonal activities, resolved conflictive situations, promoting healthy bonds, the use of authority by teachers, emotional reading, acknowledging the other person beyond cognitive variables, the ability to listen and valuing empathy in different situations with the student that affect the school setting. All of these factors depend on, among other things, the socio-emotional competence of the adult who plays a teaching role and they are linked with students' motivation in regards to learning and academic performance. The aim of this manuscript is to give a panoramic view of the current educational viewpoint, teachers' mental health, establish the importance of emotional education as well as the use of positive psychology as a paradigm in schools. Lastly, the involvement of emotional education and positive psychology in an educational setting is discussed.
\end{abstract}

Keywords: Emotional education, socio-emotional abilities, positive psychology.

\footnotetext{
${ }^{1}$ Universidad Tecnológica de Chile INACAP.

${ }^{2}$ Centro Latinoamericano de Psicología Positiva Aplicada
} 


\section{INTRODUCCIÓN}

Vivimos en una sociedad de cambio, de transformaciones permanentes, globalizante, tecnológica, conectada y desconectada, donde las modas varían tan rápido como los mensajes en una red social. Es un mundo demandante, donde la conciencia ocurre por fracciones de tiempo, nos insta a la crisis y a la reconstrucción de la realidad.

Desde el año 2006, a la fecha, la educación chilena está experimentando modificaciones a nivel político, económico y social, cobrando fuerza en la participación de jóvenes que manifestaron su descontento social con la educación impartida; por la falta de garantías para su bienestar personal y social. Esta transición no ha sido fácil, sin duda el sistema educativo es uno de los ejes que ofrece mayor resistencia al cambio, por cuanta ideología política asociada, intereses económicos, discusión de criterios y las diferentes perspectivas de los actores involucrados. Cómo plantea Milicic (2004) ... "las resistencias al cambio pueden originarse en el hecho de que vivimos en una continua contradicción emocional” ... para esclarecer esto cita a Maturana (1994), quien dice:

... "los seres modernos vivimos cada vez más en una contradicción emocional fundamental: queremos conservar el consumismo de nuestra cultura pero, al mismo tiempo, queremos conservar el mundo natural. Queremos conservar el vivir en la apropiación, pero queremos generar solidaridad; queremos certidumbre y seguridad pero, al mismo tiempo, libertad; queremos autoridad, pero también queremos mutuo respeto; queremos vivir en competencia pero, a la vez, queremos vivir en colaboración; queremos la posibilidad de hacernos infinitamente ricos, pero también queremos acabar con la pobreza; queremos ser amados, pero al mismo tiempo ser obedecidos. La vida humana no puede vivirse en armonía y dignidad si estas contradicciones emocionales no se resuelven".

Lo anterior implica comprender la cualidad inagotable que vivencian los seres humanos respecto al aprendizaje, aquello que se configura desde la controversia, que unifica la razón y la emoción, la posibilidad cierta de no dejar de aprender y reflexionar sobre temas trascendentales, como la formación de una ciudadanía más próspera, sana y feliz.

\section{Aspectos de la calidad educativa}

De acuerdo a las modificaciones establecidas por la agencia de calidad y el ministerio de educación de Chile; SIMCE no es el único indicador de calidad educativa, el documento del MINEDUC (2013)
"Otros Indicadores de Calidad Educativa” establece otras variables vinculadas al factor de "calidad", dentro de los criterios que establece el ministerio de educación. El SIMCE tendrá el valor de un 67\% y el 33\% restante será integrado por 8 indicadores que fueron aprobados por el Consejo Nacional de Educación (CNED). Los cuatro primeros medirán factores no cognitivos: autoestima académica y motivación escolar; convivencia; formación y participación ciudadana; y hábitos de vida saludable. Los otros cuatro medirán la eficiencia del sistema: tasa de asistencia y retención escolar (deserción), equidad de género y la tasa de titulación en el caso de los liceos técnicos. (MINEDUC, OTROS INDICADORES DE CALIDAD EDUCATIVA, 2014).

En desmedro de lo que representa la evaluación de estándares, el hecho de estipular criterios no cognitivos, implica un cambio en los tratos, incentivos y valor que se les da a los estudiantes. Considera demostrar altas expectativas en relación a los aprendices, tomando en cuenta sus diferencias, responsabilizándose de todos, en consecuencia, afectando positivamente su autoestima. Es curioso que 4 indicadores representativos del bienestar humano, entendiendo que su relevancia y buen funcionamiento tiene un impacto como eje preventivo de dificultades psicosociales en la institución escolar, de la deserción escolar, de formar personas más íntegras en el aspecto transversal con sentido ciudadano, corresponda a un $13,2 \%$ de lo que se entiende como calidad (MINEDUC, Manual para Establecimientos, 2014).

Durante doce años, en periodos de 8 a 10 horas los estudiantes viven en el colegio; no es azaroso decir que viven en un establecimiento educativo, pues dicha permanencia es mayor al tiempo que pasan con sus familias, debido a las distintas dinámicas sociales, necesidades económicas y demandas de un mercado laboral, que ansía en relación a la modernidad personas más calificadas y competentes. Por tanto, la escuela ratifica ser el socializador secundario por excelencia, en virtud de la permanencia de los estudiantes en dicho contexto, lo que permite en parte suplir necesidades básicas, carencias psicológicas y sociales. Sin embargo, el sistema educativo que enfrenta este estudiante, en esencia, está estructurado sobre una formación basada en la adquisición de conocimiento sostenido en sistemas de medición estandarizados (SIMCE), que pretende evaluar la adquisición de competencias básicas, para el posterior ingreso a la educación superior. En este panorama la institución escolar debe integrar y considerar aspectos relacionados con el nivel socioeconómico, capital cultural, social, psicológico y emocional de sus estudiantes, familias y comunidad escolar en general.

En relación a lo anterior, es necesario comprender la 
relevancia de los indicadores señalados, entendiendo que la institución educativa asume un rol preventivo y un factor protector frente a situaciones de riesgo a los cuales se ven expuestos los niños y adolescentes chilenos, según Halper (2010) un 22\% de trastornos mentales corresponden a niños entre los seis y los quince años. Respecto a lo anterior, la misma autora indica que la depresión adolescente es una de las causas de mayor riesgo para el intento de suicidio, aunque sus causas son diversas y muy complejas, las que van desde situaciones conflictivas en la familia, colegio, amigos o problemas relacionados con pensamientos negativos. Lo anterior, como lo indica el estudio "Panorama de Salud 2011 y sus indicadores", realizado por la OCDE, se asocia a que Chile, durante el año 2010, se ubique como el país con la segunda tasa de suicidio adolescente a nivel mundial.

\section{Panorama de la salud mental de los docentes en Chile}

El educar, en palabras simples, se relaciona con la transmisión de enseñanzas de un ser humano a otro ser humano. Esta simplicidad enfrenta una complejidad, que invita a pensar en el que enseña, quien razona, siente, convive con otros; por ende no es novedoso indicar que la violencia escolar, las dificultades salariales, la falta de valoración del propio accionar profesional, el clima laboral incida en la salud mental de los educadores y en su desempeño, afectando el de sus educandos que, a todo esto y a pesar de los distintos esfuerzos que realizan los docentes, se les evalúa y solicita resultados académicos (Cornejo, 2007).

Las normativas educativas y laborales amparan la figura docente, estableciendo parámetros para el ejercicio de la profesión y el clima laboral propicio para la actividad, sin embargo, la legislación en la práctica ofrece discrepancias, por eso llama la atención según lo establecido que, en el 1er censo docente del año 2012, donde los encuestados son 12.000 profesores, un $20 \%$ de los docentes se encuentren colegiados y un $8 \%$ participe en sindicatos de sus respectivos establecimientos. En relación a lo mencionado, Egaña y Ruiz (2013) sintetizan, en su documento "Bienestar en la Escuela, un compromiso con nuestros niños y jóvenes”, estudios asociados a la salud laboral de los docentes indican el Estudio de la Salud Laboral de los Profesores en Chile (PUC, 2003). En relación a la probabilidad de padecer burnout -esto es la respuesta al estrés laboral cuando fallan las estrategias de enfrentamiento habituales de la persona-, según este estudio el grupo de profesores experimenta en promedio más del doble de burnout $(7,8)$ que el grupo de salud primaria utilizado como referencia $(3,5)$. En síntesis, el grupo de profesores presenta mayor riesgo en bienestar psicológico y más burnout que el grupo de referencia. El informe de la UNESCO (2005) para América Latina - el que integra condiciones laborales y salud docente- establece que: un tercio de los profesores encuestados declara haber sido víctima de amenazas a su integridad física, $\mathrm{y}$ la misma proporción percibe que existen formas de violencia organizada en la escuela. En este estudio, Chile destaca tanto por su alto índice de desgaste emocional en la escala de burnout como también por tener el mayor nivel de uso de licencias médicas entre los países involucrados en la investigación. Por otra parte, un estudio realizado en el año 2011, por la Escuela de Salud Pública, de la Facultad de Medicina de la U. de Chile a 189 docentes reveló que el 30,1\% de los encuestados describieron como "regular" o "mala" su salud mental. Estudios anteriores (2008), realizados en la misma universidad, establecían que la tasa de enfermedad vinculada a la depresión y ansiedad, tríplica a la de otras profesiones. Otro estudio mencionado en el documento, de UNICEF (2005) en América Latina y España, señala que solo el $8 \%$ de los alumnos latinoamericanos y el $10 \%$ de los españoles afirman que va con placer a la escuela. Más de la mitad de los encuestados se queja de los profesores y directores de la escuela. Para comprender estos resultados cabe mencionar la explicación de Hargreaves (2005) indicada por Egaña y Ruiz (2013), quienes plantean que cuando los profesores se sienten abrumados y presionados a trabajar a gran velocidad y con fuertes exigencias ante las demandas por resultados, suelen abandonar ese trabajo más emocional e implicado con sus alumnos. Esto afecta especialmente a los docentes más comprometidos que se estresan, se desgastan y se distancian de sus propios sentimientos, tratando de hacer lo que parece ser su trabajo, llegando a adoptar actitudes cínicas, a culpar a los niños e incluso a abandonar la educación.

\section{Educación emocional y rol docente}

Desde un enfoque global, es importante señalar que el desarrollo económico, de los países, no parece ser un indicador de progreso suficiente que provea satisfacción a la ciudadanía. En este sentido no es extraño que otros países (Europa, Latinoamérica, Estados Unidos) hayan seguido el ejemplo de Bután, incorporando el ministerio de la felicidad y realizando investigaciones que permitan investigar e incrementar el bienestar y la felicidad.

Con esta mirada, es posible plantear la hipótesis de que individuos más inteligentes emocionalmente, sostienen en el tiempo mayor nivel de bienestar y salud, lo que implicaría que países que promueven este tipo de educación, potenciarían los niveles de motivación, autocontrol y bienestar en la ciudadanía, lo que potencia la calidad y productividad del recurso humano (González, 2012). 
Para lograr lo señalado anteriormente, es que se habla de la educación emocional como un eje formativo con incidencia en el desarrollo personal y en las relaciones humanas, pues las personas como individuos están sujetos al aprendizaje continuo y tener relaciones sociales a lo largo de nuestras vidas, por tanto, la educación emocional es definida como un proceso continuo y permanente que pretende potenciar el desarrollo emocional (Bisquerra, 2007). Conforme a lo estipulado, la educación emocional como instancia formativa y toda propuesta que de ella se realice, debe plasmarse con un sentido explícito en el currículo de los estudiantes, al igual que la matemática o el lenguaje, de este modo se asegura la adquisición de aprendizajes y habilidades en una trayectoria escolar, sin embargo como indica González (2012), en la amplia mayoría de las escuelas del mundo, no se dedica un minuto a la programación curricular semanal ni a actividades de educación emocional.

En relación a lo anterior, la educación emocional se establece como un factor clave para la promoción de la convivencia en el aula, en tanto que el docente debe tener educación emocional, para regular los factores interpersonales que complementan el desarrollo cognitivo y que permite, en consecuencia, la formación de una personalidad más íntegra (Galimberti, 2014), integrándose en las propuestas la adquisición de conocimientos y habilidades sobre las emociones, con el objetivo general de mejorar el bienestar individual y social.

La docencia, como profesión, debe por exposición y obligación en la interacción humana vincularse con la emoción, en este sentido Palomera (2008) establece una relación entre las emociones y habilidades citando a Brackett y Caruso, (2007) quienes indican... "Las emociones y las habilidades relacionadas con su manejo, afectan los procesos de aprendizaje, a la salud mental y física, a la calidad de las relaciones sociales y al rendimiento académico y laboral”... Palomera resalta el rol de la docencia y menciona lo expuesto por Brotheridge y Grandey, (2002) quienes señalan que... "la docencia es considerada una de las profesiones más estresantes, sobre todo porque implica un trabajo diario basado en interacciones sociales en las que el docente debe hacer un gran esfuerzo para regular no solo sus propias emociones, sino también las de los estudiantes, padres, compañeros”... Agrega además que..."Los profesores desafortunadamente, experimentan con más frecuencia emociones negativas que positivas” (Emmer, 1994)... Palomera cita ha Eysenck y Calvo, 1992, quien establece que... "las emociones negativas, como por ejemplo la ansiedad, interfieren en nuestra capacidad cognitiva para el procesamiento de la información”
... y agrega respecto a las emociones positivas, citando a Frederickson, (2001) ...”las emociones positivas, aumentan nuestra capacidad creativa para generar nuevas ideas $\mathrm{y}$, por tanto, nuestra capacidad de afrontamiento ante dificultades"... Por cuanto la importancia del rol docente y la emotividad positiva, ya que las emociones positivas de los profesores pueden aumentar el bienestar docente y también el ajuste de sus alumnos. Este afecto positivo, además, puede formar un espiral que a su vez facilite un clima de clase más favorecedor para el aprendizaje. Es por ello que la capacidad de identificar, comprender y regular las emociones, tanto positivas como negativas, se hace imprescindible en esta profesión, para poder utilizar y generar las emociones a nuestro favor. (Palomera, 2008)

Con el objetivo de sintetizar la importancia de las emociones, en la faceta interpersonal y el rol docente, Mateo (2012) explica que el arte de establecer buenas relaciones con los demás, se vincula a la habilidad de manejar emociones. Por tanto, sostiene que la empatía y la capacidad de manejar las relaciones interpersonales son cualidades imprescindibles en un aula, es por eso que no es de extrañar que la enseñanza sea una de las profesiones donde la inteligencia interpersonal se encuentre con mayor frecuencia.

El abordaje de los aprendizajes, en estas competencias, debe obedecer a niveles de complejidad y las etapas del desarrollo humano que provea entendimiento e internalización, es decir, y como indica Milicic (2013) es necesario establecer un escalonamiento de forma explícita de los aprendizajes socioemocionales, al respecto cita a Humphrey quien indica..."El aprendizaje socioemocional, definido como un proceso, sugiere un curso de acción explícito, un método o una práctica que involucra a todos los actores del establecimiento. Asimismo, el foco en la enseñanza subraya la posibilidad de sistematizar las competencias socioemocionales a desarrollar en los estudiantes, llevando a cabo un proceso de instrucción y enseñanza formal”. Por cuanto a lo anterior, subrayan la importancia del rol de los profesores en este proceso, ya que es de la mayor importancia para el desarrollo de niños y adolescentes.

\section{El docente como educador emocional}

En el contexto del desarrollo profesional docente las competencias de los profesores, para promover el aprendizaje socioemocional de sus alumnos, es uno de los elementos centrales para el logro de una formación integral de los estudiantes y está intrínsecamente ligado a la capacidad de favorecer, en los niños, el desarrollo de una identidad positiva (Aron, Milicic y Armijo, 2012). 
En relación a establecer un vínculo positivo con los estudiantes, los profesores emocionalmente competentes responden efectivamente a las necesidades de sus alumnos en tanto reconocen sus emociones, comprenden las evaluaciones cognitivas asociadas a sus emociones y entienden cómo esas cogniciones y emociones motivan la conducta de los estudiantes. Acerca del manejo de la clase, el modelo de clase prosocial sugiere que los docentes, con alta competencia socioemocional, logran una gestión efectiva en tanto promueven el entusiasmo por el aprendizaje, guían el comportamiento de los alumnos y brindan soporte a las dinámicas de conflicto al interior del aula. (Milicic, 2013)

Agregan, además, que en conjunto con estas tres variables -establecer un vínculo positivo con los alumnos, tener un manejo efectivo de la clase e implementar con éxito la enseñanza de competencias socioafectivas- se asocian con la construcción de un clima de aula nutritivo que, a su vez, influye en los resultados de los estudiantes y en la competencia y bienestar docente, creando un circuito de retroalimentación positivo. (Milicic, 2013)

Pese a lo anterior, la dimensión del desarrollo personal y educación emocional, de los docentes, está insuficientemente desarrollada o definitivamente ausente en muchos programas de formación docente inicial (Palmer, 2003). En los currículos de formación de profesores hay énfasis en lo cognitivo y en lo técnico obviando lo emocional y muchas veces tildando de un carácter poco académico, e incluso ingenuo, el trabajo en esta área (Berger, 2011).

\section{Psicología positiva y educación: desarrollo del bienestar humano}

En el ámbito educativo, sin precisar profundamente sobre aspectos teóricos, la invitación de la psicología positiva, como nuevo paradigma, es ofrecer una perspectiva de desarrollo humano que considera el desarrollo de las fortalezas, virtudes y talentos del estudiantado y de la comunidad educativa en general, a través de los distintos proyectos educativos. Este enfoque permite posicionarse desde la desigualdad humana, pues es un derecho tener educación de calidad, pero aquella calidad está supeditada al hecho de que no todos los seres humanos aprenden igual ni tienen los mismos intereses y talentos. Permitirles florecer y desarrollar habilidades personales que les permitan contrastar con el mundo que les rodea, lo cual parece el camino adecuado hacia el bienestar y la adaptación al mundo laboral.
Esta revolución educativa, centrada en el desarrollo de las fortalezas de las personas para construir y coconstruir con otro bienestar, conjuga en sintonizar la mente y el corazón. Al respecto Wachtendorff (2013) indica que la neurobiología ha acuñado el término de coherencia cardiaca el cual es un estado que se produce cuando todas nuestras partes fisiológicas están trabajando en armonía. Es un estado en que nuestros sistemas biológicos están sincronizados con el ritmo del corazón: la respiración, la digestión, la respuesta del sistema inmune, la liberación hormonal. Todos estos sistemas comienzan a sincronizarse con este creador del ritmo principal del cuerpo que es el corazón. Este estado de coherencia es el estado óptimo que facilita el aprendizaje, el rendimiento, el desarrollo y los procesos regenerativos naturales del cuerpo. Por ende, el cultivo de la atención es trascendental para poder lograr dicha integración y aquel adiestramiento es posible a través de la práctica de mindfulness o atención plena. En este aspecto Wachtendorff (2013) agrega que la actitud de atención plena sobre los estados y procesos internos es una forma de autorrelación, una forma de sintonía con uno mismo, que crea los mismos efectos que la sintonía interpersonal. En realidad, la sintonía interpersonal es una forma de compasión y la sintonía intrapersonal de la autocompasión, una forma de relacionarnos con nosotros mismos de manera empatía y comprensiva.

Respecto al desarrollo de fortalezas, tenemos las propuestas por el precursor de la psicología positiva, Martin Seligman, las cuales son veinticuatro descritas en los cuestionarios gratuitos VIA (acrónimo para Values in Action) para niños y adultos, las cuales poseen validez y confiablidad estadística a través de mediciones internacionales y su aplicabilidad es transferible a distintos ámbitos de aplicación individual y social. De la misma forma, en el ámbito organizacional, encontramos a la Gallup quienes describen treinta y cuatro fortalezas a través de sus cuestionarios pagados, relacionado a una base de datos obtenida a través de estudios en 150 países, su aplicación individual y laboral permite obtener orientación precisa respecto el desarrollo de cada una de las fortalezas y la conformación de equipos de trabajo. Esta última es interesante considerar respecto a la selección del profesorado.

Por cuanto el desarrollo de las fortalezas es posible establecer que su aprendizaje está supeditado a las propuestas educativas y al equilibrio entre lo académico y transversal, en este sentido el programa aulas felices basado en las fortalezas, propuestas por Seligman, establece cinco principios generales para crear un ambiente positivo en las escuelas, potenciar el bienestar y el aprendizaje, y favorecer 
el desarrollo de las fortalezas personales: La actitud del profesorado, el ser adultos modelos creíbles de positividad. Crear condiciones de aprendizaje que permitan fluir las experiencias de flujo se producen cuando el desafío de la tarea y las habilidades de la persona se encuentran debidamente equilibrados. Promover una educación que prime más la calidad que la cantidad, la "Educación lenta" defiende la premisa de que "menos es más en educación”; esto es: son necesarios menos aprendizajes de los que creemos, pero trabajados con mayor profundidad. Optar por modelos organizativos y metodológicos estimulantes y variados, apostar por el pluralismo metodológico, dependiendo de que lo que vayamos a enseñar es necesario optar por unas u otras formas de enseñanza. Aprovechar diversos programas ya existentes que pueden ayudar a desarrollar determinadas fortalezas personales, utilizar programas dirigidos a potenciar el desarrollo personal y social del alumnado, así como las habilidades de pensamiento.

Una faceta particular y distintiva de la psicología positiva es su rol preventivo centrado en la salud, por tanto, desglosar sus campos de acción no solo se reduce a la felicidad y la alegría; la promoción de la salud se asocia también al abordaje de temáticas como: optimismo, curiosidad y apreciación de belleza, amor por el conocimiento, desarrollo de locus de control interno, creatividad, resolución de conflictos, proyecto de vida, resiliencia, inteligencia social y emocional, entre otras áreas de intervención que, según lo establecido en las clases magistrales de Delfina Terrado (2015), deben considerar variables contextuales y culturales para su aplicabilidad en los distintos contextos escolares.

\section{CONCLUSIONES}

El docente es el actor principal del sistema escolar, por ende, su relevancia y selección debiera ser intencional, lo anterior debido a que él es el modulador de las relaciones en el aula de clase con su actitud, emocionalidad y conductas devienen consecuencias para sus estudiantes. El estilo de su pedagogía, autoritaria, democrática, laissez-faire y transformacional permitirá esclarecer el tipo de relación con sus estudiantes, su proximidad, su capacidad de empatía e involucramiento, favoreciendo el aprendizaje y desarrollo personal de los estudiantes. Este vínculo implica la necesidad de validar espacios de cuidado personal en los docentes, de forma periódica y sistemática, ya que el tratar con personas demanda el manejo de emociones propias y de otros; por tanto, ofrecer a docentes instancias de autocuidado, a través de la psicología positiva, permite empoderar positivamente respecto a su trabajo y en consecuencia mejorar su productividad y salud. En este sentido es necesario fortalecer el sistema educativo con programas preventivos orientados a la salud mental de la comunidad en general, la presencia de estas actividades permite desarrollar y fortalecer habilidades sociales y emocionales que permiten el desarrollo de la autoestima de los estudiantes y, como consecuencia, alzas en sus rendimientos escolares. A su vez permite la disminución de trastornos emocionales, consumos de drogas, embarazos prematuros y otros factores de riesgo asociados.

En tanto es urgente considerar, dentro de la formación docente, una perspectiva más humana de la educación, apostando al desarrollo de competencias socioemocionales en los docentes y asignaturas que validen el desarrollo de la educación emocional en la profesión y en la formación de los estudiantes, pues autores, como Goleman, establecen la inteligencia emocional como prioritaria, necesaria y diferenciadora del éxito en relación a la inteligencia cognitiva. Esto es validado por Pierson (2013) quien señala -como docente- que, en el ejercicio y ocaso de la profesión, la herencia más grande de un formador, cree en sus estudiantes, está ligada a la cantidad de relaciones generacionales. No es menor establecer para esta profesión que tiene carácter relacional la conclusión del estudio más largo de felicidad en sus setenta y cinco años de investigación y que continúa, el que señala: ... "las buenas relaciones mantienen la felicidad y la salud”... (Waldinger, 2015). Por lo tanto, el no reconocer este sentido, ni apuntar a la educación con una perspectiva positiva y salugénica, comprometida con el desarrollo integral es hacer un trabajo a medias y deslegitimar el valor sustantivo que tiene el colegio como unidad social y como dice Céspedes (2008) sobre algunas palabras de María Montessori... "recalcar que la educación no se centra solo en el cultivo del intelecto, sino que pone su énfasis en la formación integral del niño, es una educación que lo conduce hacia el desarrollo de una personalidad sana, sustentada en una sólida calidad interior y una voluntad de cambio en búsqueda de una sociedad mejor"... por último, hay que comprender que las instituciones educativas son promotoras de cambio y que el docente es el actor principal el cual necesita herramientas para cumplir con el objetivo trascendental: enseñar a los estudiantes a adquirir herramientas que les permitan alcanzar el bienestar. 


\section{REFERENCIAS BIBLIOGRÁFICAS}

Adserá, A. (2013). Terapia de psicología positiva. Classe R, España.

Arón, A. M. (2000). Desgaste profesional de los profesores y clima escolar. Revista Latinoamericana de Psicología, 32, $447-466$

Ricardo Arguís Rey, Ana Pilar Bolsas Valero, Silvia Hernández Paniello y M. a del Mar Salvador Monge

Arguís, R., Bolsas, A, P., Hernánde, S y Salvador, M. (2012) Programa "aulas felices" Psicología positiva aplicada a la educación. Equipo SATI. Zaragoza

Milicic, N., \& Arón, A. M. (2011). Climas sociales tóxicos y climas sociales nutritivos para el desarrollo personal en el contexto escolar. Psykhe, 9(2).

Authentic happiness, University of Pennsylvania. VIA (Values in Action), Consultado en Enero 2016. Disponible en: https://www.authentichappiness.sas.upenn.edu/es/ testcenter

Berger, C. (2014). Ponencia de Competencias Socioemocionales., (pág. 15).

Bisquerra, R. (2007). La educación emocional para la convivencia, en E. SORIANO (coord.), Educación para la convivencia intercultural. Madrid: La Muralla

Bisquerra, R. (2003). Educación Emocional y Competencias básicas para la vida. Revista de investigación educativa, 7-43.

Briceño, M. U. (2005). El liderazgo docente en la construcción de la cultura escolar de calidad: un desafío de orden superior. Prelac - Unesco, 2-11.

Cabezas-Corcione (2014). Uma introducao a la psicología positiva e suas campos de aplicacao. Annais de I Simposio LatinoBrasileiro de Psicologia Positiva.

Caruana, (2010) Aplicaciones prácticas de la psicología positiva. GENERALITAT VALENCIANA Conselleria d’Educació.

Céspedes, A. (2008). Educar las emociones, educar para la vida. Santiago de Chile: Ed. B SA.

Cornejo, R. (2008). Entre el sufrimiento individual y los sentidos colectivos: Salud laboral docente y condiciones de trabajo. Profesión Docente, 77-85.

Costa (2003), Competencia socio emocional (en línea). Fecha de consulta: octubre 2014, Disponible en: http://www.cfieavila.com/jornadas\%20salud/salud/ CompetenciaSocioEmicional.html

Christian Berger, N. M. (2009). Bienestra socio- emocional en contextos escolares: la percepción de estudiantes chilenos. Estudios sobre Educación N¹7, 21-43.

Egaña y Ruiz (2013) Bienestar en la escuela: un compromiso con nuestros niños y jóvenes.

Elvira Repetto, M. P. (2010). Las competencias socioemocionales como factor de calidad en la educación. Revista Iberoamericana sobre calidad, eficacia y cambio en educación, 83-92.
Feliz Cova, B. A. (2006). Potencialidades y obstáculos de la prevención de la depresión en niños y adolescentes. Psykhe, 15, 57-65.

Galimberti, M. (2014). Educación emocional y bienestar en ámbito académico. Revista latinoamericana de psicología positiva (PsyCap). 1, 43-51

Gallup strengths center, gallup strengths (s.f) (en línea), Consultado en Enero 2016. Disponible en: https:// www.gallupstrengthscenter.com/SignIn/GSC/ Index?workflow=100\&returnurl=/Dashboard/en-US/ Index/1

Pérez-González, J. C. (2012). Revisión del aprendizaje social y emocional en el mundo. Cómo educar las emociones, 56-69.

Goyarrola, J. M. (2011). Docentes competentes. Madrid: Narcea.

Hargreaves, A. (2003). Enseñar en la sociedad del conocimiento. Octaedro, Barcelona.

Isidora Mena, C. R. (2008). ¿Cuánto y dónde impacta? Desarrollo de habilidades socio emocionales y éticas en la escuela. Valoras UC.

Mateo, E. M. (2012). Educación emocional y habilidades sociales con alumnos con necesidades educativas especiales: propuesta de intervención. Universidad de Valladolid. Escuela Universitaria de Educación (Palencia).

Mena, I. (2008). Análisis de los OFT de convivencia y formación socioafectiva. Valoras UC.

Orrego, T. M., Milicic, N., \& Valenzuela, P. Á. (2013). Impacto en los niños de un programa de desarrollo socio-emocional en dos colegios vulnerables en Chile. Revista Iberoamericana de Evaluación Educativa, 6(2), 167-186.

MINEDUC. (2014). OTROS INDICADORES DE CALIDAD EDUCATIVA.

Myriam George, J. G. (2012). Salud mental en escuelas vulnerables: evaluación de componente promocional de un programa nacional. Revista de Psicología Universidad de Chile, 55-81.

OCDE. (2002). Resumen Ejecutivo: La definición y selección de competencias claves para la vida. DESECO, 3-19.

Ortega, C. (2010). La educación emocional y sus implicancias en la salud. REOP. 21, 462 -470.

Pérez - Escoda, B. F. (2012). Desarrollo de la competencia emocional de maestros y alumnos en contextos escolares. Electronic Journal of Research in Educational Psychology, 1184-1208.

Pierson (2013) Every kid needs a champion (en línea). Material audiovisual. Consultado en enero 2016. Disponible en: http://www.ted.com/talks/rita_pierson_ every_kid_needs_a_championRadioUchile (2011) Según informe OCDE: "Chile se pone colorado porque la tasa de suicidios es una de las más altas del mundo" (en línea). Reportaje online. Consultado en Enero 2014. Disponible en: http://radio.uchile.cl/2011/11/28/ 
manalich-chile-se-pone-colorado-porque-la-tasa-desuicidios-es-una-de-las-mas-altas-del-mundo

Palomera, R., Fernández-Barrocal, P., Brackett, M. (2008). Inteligencia Emocional como una competencia básica en la formación inicial de los docentes. Education and Psychology. 6 (2), 437-454.

Seligman, M. E. (2014). Florecer: La nueva psicología positiva y la búsqueda del bienestar. Editorial Oceano.
Tamara Otzen, A. S. (2014). Mortalidad por suicidio en Chile: tendencias en los años 1998 - 2011. Revista Médica Chile, 305-313.

Wachtendorff, H (2013) "Coherencia cardiaca", trabajo final consultor en mindfulness, 7 - 10 .

Waldinger(2015), What makes a good life lessons from the longest study on happiness (en línea). Material audiovisual. Consultado en enero 2016. Disponible en: http://www.ted.com/talks/robert_waldinger_what_ makes_a_good_life_lessons_from_the_longest_study_ on_happiness?language $=\mathrm{en}$ 